\title{
Secret Admirer
}

\author{
Jason Lie
}

Before you call the police or stab me with your knife know that I'm just here to

Crawl into your bedroom so I can sweep your fallen curls and knit myself a scarf

Tiptoe into your bathroom so I can pile your dandruff sprinkles and glue them to my ceiling

Slink into your laundry room so I can bag your dirty gym clothes and bath in the wringed out sweat

Dive into your rubbish bin so I can rummage for your nails clippings and pour them into my maracas

Before you call the police or stab me with your knife know that I love you. 